\title{
Spin-dependent transport in a two-dimensional GaAs electron gas in a parallel magnetic field
}

\author{
Yu-Ming Cheng ${ }^{\mathrm{a}}$, Tsai-Yu Huang ${ }^{\mathrm{a}}$, Chao Han $\mathrm{Pao}^{\mathrm{a}}$, Chun-Cheng Lee ${ }^{\mathrm{a}}$, C.-T. Liang ${ }^{\mathrm{a}, *}$, \\ M.Y. Simmons ${ }^{b}$, C.G. Smith ${ }^{c}$, D.A. Ritchie ${ }^{c}$, M. Pepper ${ }^{c}$, Gil-Ho Kim ${ }^{d}$, J.Y. Leem ${ }^{\mathrm{e}}$ \\ ${ }^{a}$ Department of Physics, National Taiwan University, Taipei 106, Taiwan \\ ${ }^{\mathrm{b}}$ School of Physics, University of New South Wales, Sydney 2052, Australia \\ ${ }^{\mathrm{c}}$ Cavendish Laboratory, Madingley Road, Cambridge CB3 OHE, UK \\ d Telecommunication Basic Research Laboratory, ETRI, Yusong P. O. Box 106, Taejon 305-600, South Korea \\ ${ }^{\mathrm{e}}$ Materials Evaluation Centre, KRISS, P.O. Box 102, Taejon 305-600, South Korea
}

\begin{abstract}
We report low-temperature magnetoresistivity measurements of high-quality gated two-dimensional (2D) electron systems. In the dilute electron density limit, we show evidence for spin polarisation in an in-plane magnetic field. Using a simple model, we estimate the Landé $g$-factor in this dilute two-dimensional electron gas to be about 3.32. The enhanced Landé $g$-factor compared with that of a bulk GaAs $2 \mathrm{D}$ electron system $(0.44)$ is ascribed to electron-electron interaction effects at ultra-low electron densities. (C) 2002 Elsevier Science B.V. All rights reserved.
\end{abstract}

PACS: 73.40.Gk; 73.20.Dx

Keywords: Spin; $g$-factor; Parallel magnetic field

In a low-dimensional electron system [1], applying an in-plane magnetic field $B_{\|}$parallel to the plane of the electron gas has been proved to be a powerful technique to study spin-dependent electron transport. The first tilted magnetic field experiment on a two-dimensional electron gas (2DEG) revealed an enhancement of the $g$-factor [2]. In a one-dimensional (1D) electron system, it was first demonstrated by Wharam et al. [3] that a large $B_{\|}$lifts the electron spin-degeneracy, causing consecutive spin-parallel (parallel to $B_{\|}$) and spin-antiparallel (anti-parallel to $\left.B_{\|}\right)$conductance plateaux in multiples of $e^{2} / h$ [4].

\footnotetext{
* Corresponding author. Fax: +886-2-23639984.

E-mail address: ctliang@phys.ntu.edu.tw (C.-T. Liang).
}

Using a source-drain bias technique [5], Patel et al. [4] measured the Landé $g$-factor in a one-dimensional (1D) constriction for the first time. Later this measurement was extended to the case of an ultra-high-quality $1 \mathrm{D}$ electron gas. When the $1 \mathrm{D}$ channel is wide, it is found that the measured Landé $g$-factor is $\approx 0.4$, close to that of bulk GaAs. As the channel is progressively narrowed there is an enhancement of the Landé $g$-factor [6].

Recently there has been a great deal of interest in charge transport in dilute 2D systems [7-10]. In low carrier concentrations there is a significant drop in the resistance with decreasing temperature. It is also found that application of an in-plane magnetic field remove this increase and as the effect is not dependent on direction of field it seems most likely to be associated 
with spin $[10,12,13]$. A change in density of states could be expected to influence the screening properties of the dilute system as well as any possible interaction between them.

Previously we studied spin-dependent electron transport in a quasiballistic quantum wire [14]. It was found that at zero split-gate voltage when the quasiballistic wire is not electrostatically defined, the two-terminal conductance due to the bulk 2DEG shows monotonic decrease with increasing $B_{\|}$. In order to gain more insights into the effect of an $B_{\|}$on 2DEG transport and exclude any contact resistance effect, we measure the four-terminal magnetoresistivity of a gated 2D electron system at various carrier densities. In this paper, we report low-temperature magnetoresistivity measurements of $2 \mathrm{D}$ GaAs electron gases in which carrier-carrier interactions are much weaker compared with those in a GaAs hole gas [10] and in a Si electron gas [11]. Our experimental results fall into two categories. In the dilute density limit, we shall show evidence for spin polarisation in an in-plane magnetic field. Using a simple model, we estimate the Landé $g$-factor in this dilute $2 \mathrm{DEG}$ to be about 3.32. The enhanced value of the Landé $g$-factor in this dilute limit compared with that of a bulk 2DEG (0.44) is ascribed to electron-electron interactions and over the whole measurement range $r_{s}$ does not vary significantly.

The devices used in this work are two gated Hall bars made from $\mathrm{GaAs} / \mathrm{Al}_{0.33} \mathrm{Ga}_{0.67} \mathrm{As}$ heterostructure. Sample A has a carrier density of $1.4 \times 10^{15} \mathrm{~m}^{-2}$ with a mobility $\mu$ of $400 \mathrm{~m}^{2} \mathrm{~V} \mathrm{~s}^{-1}$ at $V_{\mathrm{g}}=0$ after brief illumination with a red light-emitting diode. Sample $\mathrm{B}$ has a 2 DEG carrier density of $3.3 \times 10^{15} \mathrm{~m}^{-2}$ and a mobility of $30 \mathrm{~m}^{2} \mathrm{~V} \mathrm{~s}^{-1}$ at $V_{\mathrm{g}}=0$ without illumination. Experiments were performed in a top-loading ${ }^{3} \mathrm{He}$ cryostat at $T=300 \mathrm{mK}$ and the four-terminal magnetoresistivity was measured with standard phase-sensitive techniques. The in-plane magnetic field $B_{\|}$is applied parallel to the source-drain current. To check for an out-of-plane magnetic field component, we measure the Hall voltage. From this we know that the sample was aligned better than $0.1^{\circ}$ using an in-situ rotating insert.

Fig. 1 shows the four-terminal magnetoresistivity $\rho_{x x}$ as a function of in-plane magnetic field $B_{\|}$at various carrier densities $n_{s}$. Let us consider the uppermost curve. It is evident that $\rho_{x x}$ shows a $B_{\|}^{2}$ dependence

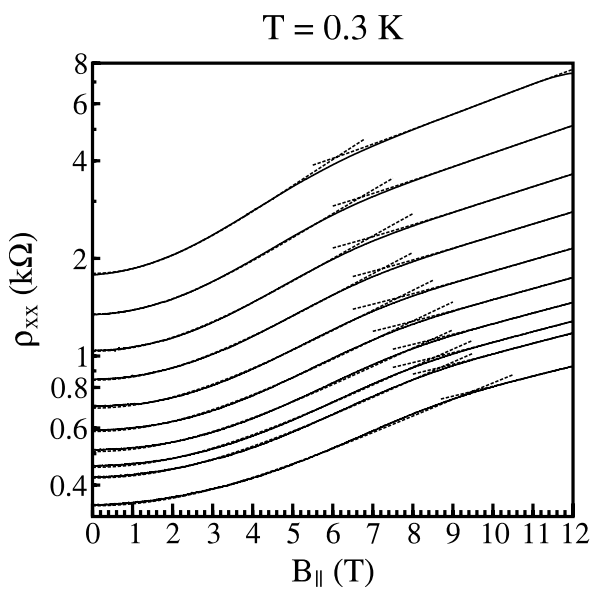

Fig. 1. $\rho_{x x}\left(B_{\|}\right)$for various carrier densities. From top to bottom: $n_{s}=1.379,1.481,1.591,1.688,1.780,1.884,1.967,2.036$, 2.076 and $2.226 \times 10^{10} \mathrm{~cm}^{-2}$, respectively. Two parabolic fits for $B_{\|}<5 \mathrm{~T}$ and $B_{\|}>9 \mathrm{~T}$ for various $n_{s}$ are shown in dotted lines. The data is taken on Device A.

for $B_{\|}<5 \mathrm{~T}$ and shows a weaker $B_{\|}^{2}$ dependence for $B_{\|}>9 \mathrm{~T}$, as shown by the two dotted lines. We ascribe the increase in $\rho_{x x}$ at low $B_{\|}$to gradual spin alignment of the $2 \mathrm{DEG}[10,11]$. It is worth mentioning that in both previous work $[10,11], \rho_{x x}$ shows an exponential $B$ dependence in both low and high magnetic field regimes. We believe the fact that in our case $\rho_{x x}$ shows a $B^{2}$ dependence is due to much weaker carriercarrier interactions compared with those in previous studies $[10,11]$. The physical origin of $B_{\|}^{2}$ at high $B_{\|}$ is believed to be due to the enhancement of electron scattering when the magnetic length is comparable to the thickness of the 2DEG [12]. This is supported by the fact that at $B_{\|}=9 \mathrm{~T}$ where $\rho_{x x}$ starts showing a weak $B^{2}$ dependence, the corresponding magnetic length is $\approx 9 \mathrm{~nm}$, in close agreement with a typical 2DEG thickness of $10 \mathrm{~nm}$. To obtain quantitative information on this spin alignment effect, we use an empirical method similar to those reported [10,11], but using two parabolic fits, as shown in the two dotted lines in Fig. 1 for various $n_{s}$. The interception of two parabolic fits is defined as the "crossing field" $B_{\text {cross }}$ for a certain 2D carrier density. As shown later, from $B_{\text {cross }}\left(n_{s}\right)$ we can estimate the $g$-factor in our system. Note that the magetoresistivity shows little temperature dependence between $T=0.3 \mathrm{~K}$ and $1 \mathrm{~K}$, suggesting that the $B_{\|}^{2}$ dependence is a semi-classical effect. 


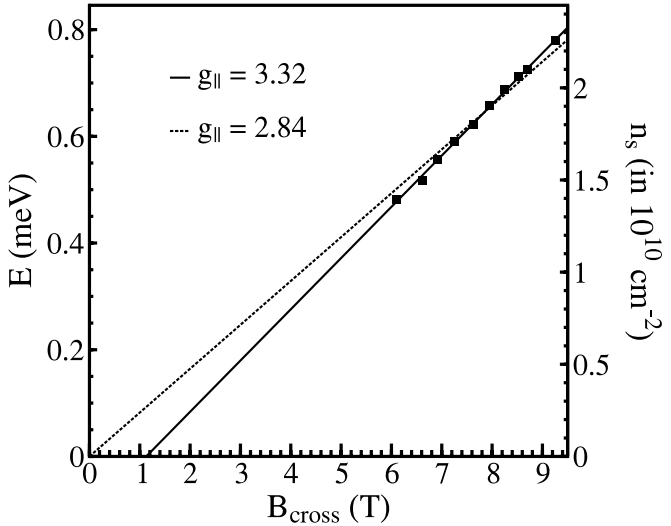

Fig. 2. Local Fermi energy $E$ and the corresponding 2D carrier density $n_{s}$ at various measured crossing field $B_{\text {cross }}$. The straight line fit through the origin is shown in the dotted line. The best linear fit is shown in the solid line.

Also within our experimental accuracy, we did not observe a "metal-insulator transition" [15] in our device.

Fig. 2 shows the crossing field $B_{\text {cross }}$ as a function of both carrier concentration $n_{s}$ and the corresponding local Fermi energy $E$. Following the previous work $[10,11]$, we assume the slope of the $E-B_{\text {cross }}$ diagram is given by the Zeeman energy $E=1 / 2 g \mu_{\mathrm{B}} B_{\|}$, where $\mu_{\mathrm{B}}$ is the Bohr magneton. In this case, a linear fit through the origin gives an estimated $g$-factor of 2.84 . As shown in Fig. 2, the best linear fit yields a value of the $g$-factor of 3.32. This fit gives a negative interception at $B=0$ which can be attributed to disorder broadening [11]. Note that both measured values are close to that measured in a clean 1D electron gas when there is a single 1D subband occupied [6]. Previously this enhancement of $g$-factor [6] is ascribed to electron-electron interactions at low carrier densities. We note that the dimensionaless parameter $r_{s}$, the ratio of the Coulomb interaction energy to the kinetic (Fermi) energy reflects the strength of electronelectron interactions in the system. In our system, $r_{s}$ is $\approx 4.7$ at the lowest carrier density and decreases to 3.7 at the highest $n_{s}$. Therefore, over the whole measurement range, $r_{s}$ only decreases by an amount of $\approx 20 \%$. In this case, we believe that the strength of electronelectron interactions does not vary significantly over the whole measurement range, thus giving rise to an approximately constant $g$-factor determined from the straight line fit shown in Fig. 2.

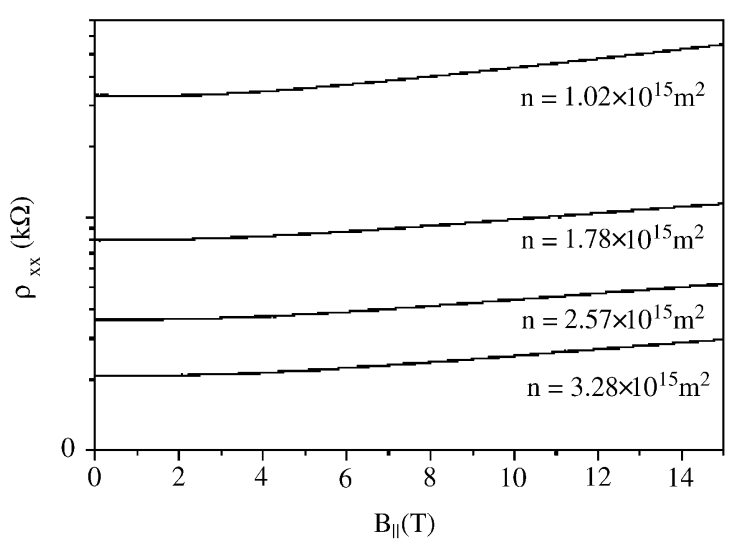

Fig. 3. Four-terminal resistivity $\rho_{x x}$ as a function of $B_{\|}$at four different carrier densities. The data is taken on Device B.

We now turn our attention to in-plane field measurements in the high carrier density limit. Fig. 3 shows the four-terminal resistivity $\rho_{x x}$ as a function of in-plane magnetic field at four different carrier densities. It is evident that with increasing $B_{\|}$, the resistivity gradually increases. With the attainable magnetic field, we are not able to observe saturation of $\rho_{x x}$ in a high parallel magnetic field. Thus, we cannot estimate the $g$-factor in our system. It would be interesting to measure the in-plane $g$-factor in the high-density limit in which electron-electron interactions are weak in the system.

In conclusion, we have measured gated 2D GaAs electron gases. In the dilute electron density limit, our experimental results show that the magnetoresistance exhibits a much weaker $B_{\|}^{2}$ dependence compared with those in a GaAs hole gas and in a Si electron system. Using an empirical method, we estimate the Landé $g$-factor to be 3.32 in this dilute GaAs 2DEG. This enhanced $g$-factor is ascribed to electron-electron interactions and the fact that over the whole measurement range $r_{s}$ does not vary significantly. In the high density limit, parallel magnetic field measurements also show a gradual enhancement of the magnetoresistivity.

This work was funded by the NSC, Taiwan. The low-temperature measurements were performed at the high-magnetic field facilities at the Centre for Condensed Matter Sciences (CCMS), National Taiwan University (NTU). The work at Cambridge was supported by the UK EPSRC. We thank H.H. Cheng at 
the CCMS, NTU for invaluable experimental help. G.H.K. is grateful for financial support from the Korean MIC.

\section{References}

[1] A.B. Fowler, F.F. Fang, W.E. Howard, P.J. Stiles, Phys. Rev. Lett. 16 (1996) 901.

[2] F.F. Fang, P.J. Stiles, Phys. Rev. 174 (1968) 823.

[3] D.A. Wharam, T.J. Thornton, R. Newbury, M. Pepper, H. Ahmed, J.E.F. Frost, D.G. Hasko, D.C. Peacock, D.A. Ritchie, G.A.C. Jones, J. Phys. C 21 (1988) L209.

[4] N.K. Patel, J.T. Nicholls, L. Martin-Moreno, M. Pepper, J.E.F. Frost, D.A. Ritchie, G.A.C. Jones, Phys. Rev. B 44 (1991) R10 973.

[5] N.K. Patel, J.T. Nicholls, L. Martin-Moreno, M. Pepper, J.E.F. Frost, D.A. Ritchie, G.A.C. Jones, Phys. Rev. B 44 (1991) 13549.
[6] K.J. Thomas, J.T. Nicholls, M.Y. Simmons, M. Pepper, D. Mace, D.A. Ritchie, Phys. Rev. Lett. 77 (1996) 135.

[7] D. Simonian, S.V. Kravchenko, M.P. Sarachik, V.M. Pudalov, Phys. Rev. Lett. 79 (1997) 2304.

[8] M.Y. Simmons, A.R. Hamilton, M. Pepper, E.H. Linfield, P.D. Rose, D.A. Ritchie, A.K. Savchenko, T.G. Griffiths, Phys. Rev. Lett. 80 (1998) 1292.

[9] T. Okamoto, K. Hosoya, S. Kawaji, A. Yagi, Phys. Rev. Lett. 82 (1999) 3875.

[10] J. Yoon, C.C. Li, D. Shahar, D.C. Tsui, M. Shayegan, Phys. Rev. Lett. 84 (2000) 4421.

[11] V.M. Pudalov, G. Brunthaler, A. Prinz, G. Bauer, Cond-mat/0004206.

[12] S. Das Sarma, E.H. Hwang (1999) Cond-mat/9909452.

[13] V.T. Dolgopolov, A. Gold, JETP Lett. 71 (2000) 27.

[14] C.-T. Liang, M. Pepper, M.Y. Simmons, C.G. Smith, D.A. Ritchie, Phys. Rev. B 61 (2000) 9952.

[15] Y. Hanein, D. Shahar, J. Yoon, C.C. Li, D.C. Tsui, H. Shtrikman, Phys. Rev. B 58 (1998) 13338. 